\title{
Avaliação clínica e radiográfica do joelho de cães submetidos à cirurgia para correção da ruptura do ligamento cruzado cranial: estudo retrospectivo de três anos
}

\section{Clinical and radiographic evaluation of canine stifle joint after surgical stabilization for the treatment of cranial cruciate ligament rupture: retrospective study of three years}

\author{
Kalinne Stephanie Bezerra ${ }^{1 *}$; Fernando De Biasi ${ }^{2}$
}

\begin{abstract}
Resumo
O presente trabalho objetivou avaliar clínica e radiograficamente o joelho de cães com até dois anos e meio de pós-operatório da correção da ruptura de ligamento cruzado cranial. Realizou-se um estudo retrospectivo de três anos dos prontuários dos animais atendidos no Hospital Veterinário da Universidade Estadual de Londrina, submetidos a esta cirurgia no período compreendido entre fevereiro de 2008 a fevereiro de 2011. Totalizando 28 casos e 33 joelhos, nos quais foram realizadas diferentes técnicas cirúrgicas para a estabilização articular. A prevalência foi de fêmeas, média de idade superior a sete anos e cães com peso acima de $25 \mathrm{~kg}$. Oitenta e dois por cento dos animais apresentaram melhora clínica satisfatória, independente da técnica cirúrgica utilizada. Apesar da progressão da artrose e da persistência de discreto movimento de gaveta no pós-operatório, não houve diferença clínica evidente com relação ao grau de claudicação. Animais mais leves apresentaram prognóstico melhor. Não ocorreu diferença significativa entre as técnicas cirúrgicas com relação aos parâmetros avaliados neste período pós-operatório.
\end{abstract}

Palavras-chave: Ortopedia, cirurgia, ruptura de ligamento cruzado cranial, cães

\begin{abstract}
This study aimed a clinical and radiographic evaluation of stifle joint of dogs with up to two and a half years of postoperative correction of cranial cruciate ligament rupture. A retrospective study of three years has been done, based on the animals files treated at the Hospital Veterinário da Universidade Estadual de Londrina, underwent this surgery during the period from February 2008 to February 2011. Totalizing 28 cases and 33 stifle joints, which were performed different surgical techniques for joint stabilization. The prevalence was female, middle aged over seven years and dogs weighing over $25 \mathrm{~kg}$. Eighty-two percent of the animals showed satisfactory clinical improvement, independent of surgical technique. Despite the progression of arthrosis and the persistence of discrete drawer movement in the postoperative period, no evident clinical differences were noted in relation to lameness. Lighter animals showed better prognosis. There was no evident difference between the surgical techniques in relation to the parameters evaluated in the postoperative period.
\end{abstract}

Key words: Orthopedy, surgery, cranial cruciate ligament rupture, dogs

Discente, Universidade Estadual de Londrina, UEL, Londrina, PR. E-mail: kalinne_stephanie@yahoo.com.br

Prof. Dr. do Dept ${ }^{\circ}$ de Clínicas Veterinárias, CCA, UEL, Londrina, PR. E-mai: biasif@yahoo.com

Autor para correspondência 


\section{Introdução}

Os pacientes com problemas ortopédicos têm importância significativa dentro da clínica geral e dentre esses, as queixas de afecções articulares estão entre as mais frequentes (JOHNSON, 2008). A ruptura do ligamento cruzado cranial é a enfermidade mais comum do joelho canino, sendo esta uma das principais causas de claudicação em membros pélvicos (BENNETT, 1990; VASSEUR, 2007).

O ligamento cruzado cranial tem por função limitar o deslocamento cranial da tíbia em relação ao fêmur, impedir a rotação interna da tíbia em relação ao mesmo e evitar a hiperextensão do joelho. Trauma, degeneração ligamentar em cães idosos e presença de conformação anormal da articulação do joelho estão entre as principais causas de ruptura (BUTTERWORTH; DENNY, 2000; SCHULZ, 2008; HULSE, 1995). Porém considera-se que esta seja uma afecção com diferentes etiologias primárias, pois cães de qualquer idade, raça, estado reprodutivo, tamanho, condição corporal e função podem ser acometidos (COOK, 2010a).

O sinal clínico mais evidente da ocorrência de ruptura do ligamento é a claudicação, seja ela súbita, que é normalmente associada a trauma, ou intermitente seguida de piora progressiva. Pode ocorrer também uma ruptura parcial, onde há claudicação leve por vários meses, até que ocorra por fim a ruptura total (BUTTERWORTH; DENNY, 2000; SCHULZ, 2008). Ao exame físico, o animal pode ou não apresentar dor, efusão articular, hipotrofia muscular do membro acometido e crepitação, ocorrendo variações de acordo com o histórico do paciente (SCHULZ, 2008).

O diagnóstico definitivo é feito pelo teste de gaveta positivo, no qual há deslocamento cranial da tíbia em relação ao fêmur, movimento que não seria possível se o ligamento estivesse íntegro (SCHULZ, 2008). O exame radiográfico usualmente não tem caráter diagnóstico e é utilizado para excluir outras possíveis causas de claudicação e avaliar o grau de doença articular degenerativa assim como sua progressão (BUTTERWORTH; DENNY, 2000; SCHULZ, 2008).

Quanto ao tratamento, a correção cirúrgica é indicada, principalmente quando se trata de animais acima de $15 \mathrm{~kg}$. A cirurgia visa à estabilização da articulação do joelho e à redução da velocidade de progressão da doença articular degenerativa (HULSE, 1995; VASSEUR, 2007).

As modalidades cirúrgicas correspondem às técnicas intracapsulares, extracapsulares e osteotomias tibiais. Segundo a literatura, nenhum dos procedimentos se mostrou claramente superior aos demais (VASSEUR, 2007), todos apresentando resultados similares e satisfatórios, sendo a escolha da técnica apenas uma questão da preferência do cirurgião (BUTTERWORTH; DENNY, 2000; HULSE, 1995).

As técnicas extrarticulares vêm ganhando cada vez mais destaque devido à certas vantagens, das quais se pode citar uma maior segurança do procedimento, relativa facilidade técnica, pouco requerimento de material e baixo custo. A principal desvantagem é a de que o fio pode estirar ou romper em algum ponto após ter sido implantado, proporcionando certa instabilidade ou disfunção (COOK, 2010b).

A técnica extracapsular com pontos isométricos proporciona um menor desgaste do fio, teoricamente proporcionando uma maior estabilidade articular por um período maior de tempo. Atualmente tem sido associada com um maior índice de segurança, sendo considerada uma técnica que proporciona resultados iguais ou melhores que os outros métodos de correção da ruptura do ligamento cruzado cranial (COOK, 2010b).

Vários estudos demonstram que não há diferença entre as técnicas adotadas (VASSEUR, 2007) e a progressão de artrose continua independentemente da realização da correção cirúrgica (VASSEUR; BERRY, 1992). 
Este estudo objetiva uma análise retrospectiva dos casos atendidos no Hospital Veterinário da Universidade Estadual de Londrina, comparando-se os resultados obtidos com os conhecimentos prévios sobre o assunto, a fim de verificar se há correlação entre o prognóstico e determinada técnica cirúrgica, ou seja, qual tratamento se mostra mais eficaz.

\section{Materiais e Métodos}

Foi realizado um levantamento de dados do total de cirurgias ortopédicas realizadas no Hospital Veterinário da Universidade Estadual de Londrina no período de fevereiro de 2008 a fevereiro de 2011, dos quais foram analisados 28 prontuários de cães que foram submetidos à cirurgia para correção de ruptura do ligamento cruzado cranial.
Os dados obtidos foram: sexo, raça, peso, idade, grau de claudicação pré-operatória, tempo entre o início dos sinais clínicos e o diagnóstico, afecções ortopédicas associadas e técnica cirúrgica utilizada. Posteriormente, agendou-se um retorno com os proprietários para avaliação ortopédica obtendo-se o grau de claudicação, intensidade do movimento de gaveta e exame radiográfico do joelho. O grau de claudicação foi determinado em uma escala de 0 a 10, sendo zero a ausência de claudicação e dez a impotência funcional do membro, de acordo com o Figura 1. A intensidade do movimento de gaveta foi classificada de grau 0 a 3, sendo: 0 , a ausência de movimento de gaveta; 1 , gaveta de intensidade leve; 2 , moderada e 3 , grave.

Tabela 1. Grau de claudicação em cães.

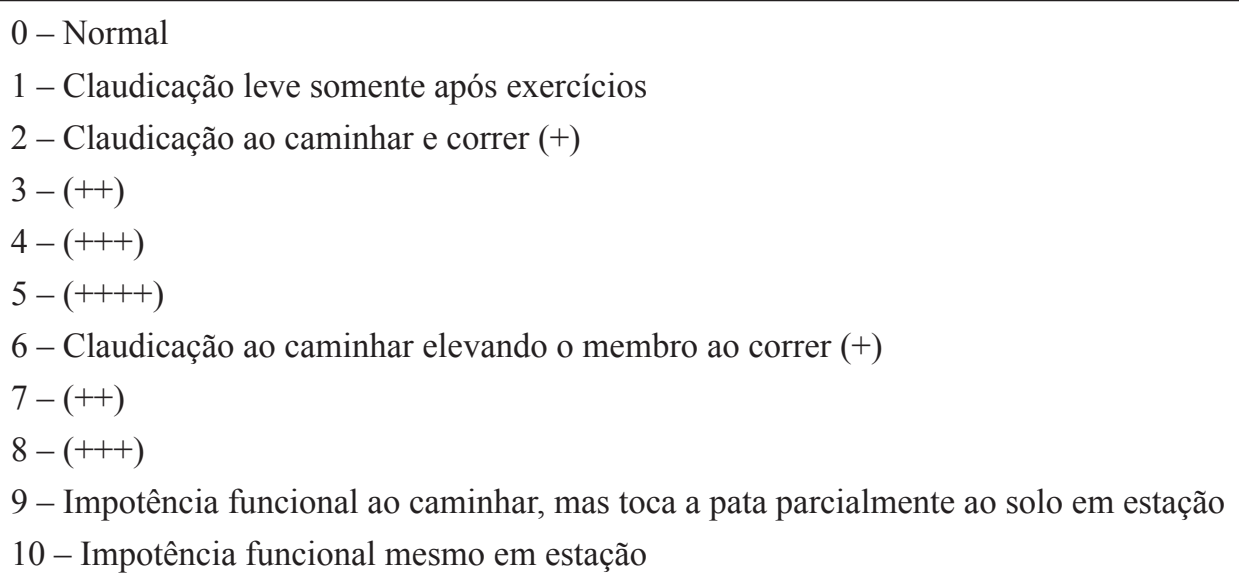

Fonte: Hospital Veterinário da Universidade Estadual de Londrina. Elaboração dos autores.

As radiografias foram avaliadas quanto à intensidade da artrose em dois momentos (pré e pósoperatório). Utilizou-se um sistema de escore para pontuação de 18 alterações radiográficas, sendo elas: 1) lesão subcondral radiolucente no sulco da tróclea femoral, 2) reação periosteal no aspecto proximal do sulco da tróclea femoral, 3) perda da definição do trabeculado ósseo patelar, 4) osteófito no aspecto apical da patela, 5) osteófito no aspecto basilar da patela, 6) enteseófito no côndilo femoral lateral, 7) enteseófito no côndilo femoral medial, 8) osteófito no côndilo tibial lateral, 9) irregularidade na superfície articular caudal da tíbia, 10) lesão radiolucente no côndilo tibial medial, 11) osteófito no côndilo tibial medial, 12) osteófito no aspecto caudal da tíbia, 13) reação periosteal no aspecto 
proximal e face medial da tíbia, 14) osteófito na eminência intercondilar da tíbia, 15) lesão subcondral radiolucente no aspecto central da tíbia, 16) reação periosteal no sesamóide do gastrocnêmio medial, 17) reação periosteal no sesamóide do poplíteo, 18) fragmento ósseo mineralizado intraarticular. Cada alteração radiográfica foi graduada com um escore de acordo com a sua gravidade, sendo 0 (ausente), 1 (leve), 2 (moderada) e 3 (grave). Baseado no método de Vasseur e Berry (1992), a soma dos escores atribuídos para cada alteração presente indicou a intensidade de artrose para cada animal. Todos os dados foram analisados de forma descritiva.

\section{Resultados e Discussão}

De fevereiro de 2008 a fevereiro de 2011, foram realizadas 291 cirurgias ortopédicas no Hospital Veterinário da Universidade Estadual de Londrina, dentre as quais, 58 foram procedimentos efetuados em joelhos de cães, o que representa uma casuística de aproximadamente $20 \%$ do total de cirurgias ortopédicas desses três anos.

No estudo, foram analisados 28 casos de animais que foram submetidos à cirurgia para correção da ruptura de ligamento cruzado cranial, sendo 34 joelhos ao todo, com período de avaliação pósoperatória que variou de 30 dias a dois anos e 141dias.

Dos casos estudados, aproximadamente 64\% dos cães eram fêmeas e $36 \%$ machos. Observouse uma prevalência de fêmeas em relação a machos, o que condiz com literatura, que relata em alguns trabalhos uma incidência maior em fêmeas (HARASEN, 1995, 2003).

O peso destes animais variou de 3 a $40,5 \mathrm{~kg}$, sendo 10 cães abaixo de $15 \mathrm{~kg}$ e 18 acima de $25 \mathrm{~kg}$. A idade variou de um a 13 anos, onde 16 animais tinham idade superior a sete anos. Estes dados estão de acordo com a literatura, que demonstra que apesar da ruptura afetar cães de qualquer raça e qualquer idade, a maior incidência é em raças de grande porte e em animais com idade mais avançada (WHITEHAIR; VASSEUR; WILLITS, 1993).

A média de idade dos animais que tiveram abaixo de $15 \mathrm{~kg}$ foi de 7,1 anos, enquanto que a dos que tiveram acima de vinte e cinco quilos foi de 6, 2 anos e dos 28 cães, 21 apresentaram idade superior a cinco anos. Corrobora o fato de que um dos fatores para que haja a ruptura do ligamento cruzado cranial é a degeneração ligamentar que ocorre com o avanço da idade. Esse processo é mais precoce em cães mais pesados, acima de $15 \mathrm{~kg}$, ao contrario do que acontece com cães mais leves onde esta degeneração ocorre mais tardiamente (JOHNSON; JOHNSON, 1993).

Quatro cães já apresentavam ruptura do ligamento cruzado cranial bilateral no momento do diagnóstico, e em dois houve ruptura no membro contralateral algum tempo após a primeira cirurgia do joelho. Este fato é descrito na literatura, que relata a incidência de ruptura no membro contralateral em 10 a 25\% dos casos em até três anos (JOHNSON; JOHNSON, 1993).

Dos 34 joelhos analisados, nove foram submetidos à técnica extracapsular nos pontos isométricos e fio poliéster sem artrotomia e outros dois receberam a mesma técnica, porém com artrotomia; cinco receberam a técnica extracapsular nos pontos isométricos e fio de náilon sem artrotomia, e cinco com artrotomia; três, a técnica intracapsular Paatsama; cinco, a técnica extracapsular com fio de náilon sem artrotomia e três, com artrotomia; um cão recebeu a técnica intracapsular Paatsama associada à técnica extracapsular com fio de náilon.

Dos 33 joelhos, 24 apresentaram, no período pós-operatório, intensidade de movimento de gaveta discreto (Grau 1) e em sete houve movimento de gaveta de intensidade moderada (Grau 2), independentemente da técnica adotada. Um estudo comparativo in vitro entre as técnicas extrarticulares demonstrou que houve alguma intensidade de movimento de gaveta mesmo após 
a correção, em dias ou semanas após a cirurgia (BOTTECHER; GREVEL; WERNER apud HARASEN, 2007). Esse resultado demonstra que nenhuma técnica consegue restaurar, em longo prazo, a biomecânica equivalente à do ligamento original, bem como, não há diferença nos resultados clínicos entre elas (SMITH, 2000).

Dos 28 casos, 23 apresentaram melhora clínica satisfatória da claudicação (Grau 0, 1 e 2) independente da técnica cirúrgica utilizada, representando $82 \%$ dos animais que foram submetidos à cirurgia para correção da ruptura do ligamento cruzado cranial. Segundo Moore e Read (1995), independentemente da técnica cirúrgica, 85,7 a $91 \%$ dos animais apresentam melhora clínica satisfatória após a cirurgia. Vale ressaltar que dois animais sofreram trauma por acidentes e tiveram que re-operar o joelho, um dos quais sofreu um corte na região por arame de cerca, 72 dias após a cirurgia e o outro foi atropelado 1 ano e 29 dias após, rompendo novamente o ligamento, porém apresentava grau 0 de claudicação anterior ao acidente.

Dos 28 cães, sete melhoraram completamente da claudicação com média de 90 dias pós-operatórios, com a técnica extrarticular, independente do fio, com ou sem artrotomia. O peso destes animais variou de 3 a vinte e cinco quilos, dentre os quais apenas dois tinham acima de $10 \mathrm{~kg}$. Todos esses cães apresentavam discreto movimento de gaveta (Grau 1) à reavaliação. Neste trabalho, $25 \%$ dos animais não apresentaram claudicação ao final do período de avaliação, o que concorda com a literatura no fato de que a completa recuperação ocorre em menos de $50 \%$ dos animais (MOORE; READ, 1996). Isso também demonstra que cães mais leves têm um prognostico melhor quanto ao retorno da função normal do membro.

Um estudo comparativo, realizado em cães da raça labrador retriever, avaliando o efeito de diferentes modalidades de técnicas cirúrgicas para estabilização do joelho sobre a função do membro, demonstrou que apenas $15 \%$ dos animais que receberam as técnicas, extra e intracapsulares, não podiam ser distinguidos clinicamente de cães normais após seis meses de pós-operatório. Melhora significativa foi vista em $40 \%$ dos cães que receberam a técnica extrarticular (CONZEMIUS et al., 2005).

Outros sete cães apresentaram claudicação discreta somente após exercício (Grau 1) ao período final de avaliação, que variou de 43 dias a 2 anos e 85 dias. O peso destes cães variou de 5,7 a 40, 5 $\mathrm{kg}$, dentre os quais apenas três tinham abaixo de 25 $\mathrm{kg}$. Esses cães apresentaram discreto movimento de gaveta (Grau 1), sendo que cinco deles receberam a técnica extrarticular, independentemente do fio, com ou sem artrotomia, e dois a técnica de Paatsama. Independentemente da técnica utilizada, estudos mostram que a maioria dos cães ainda persiste com algum grau de claudicação intermitente após a cirurgia (MOORE; READ, 1996).

Nove cães apresentaram ao período final de avaliação, que variou entre 14 dias e dois anos, claudicação de grau leve (Grau 2), movimento de gaveta discreto (Grau 1), peso que variou entre 5 e 40 $\mathrm{kg}$, sendo que sete desses nove tinham acima de 30 $\mathrm{kg}$. Dos nove casos, três foram submetidos à técnica Paatsama e técnicas extracapsulares, com náilon ou poliéster, com ou sem artrotomia. Todos esses cães tinham doenças ortopédicas intercorrentes. $\mathrm{O}$ prognóstico se torna mais reservado quanto maior o peso do cão e com a presença de doenças ortopédicas concomitantes a ruptura do ligamento.

A vantagem da técnica extrarticular mantendo-se os pontos isométricos do joelho seria a manutenção da mesma distancia entre os locais de fixação dos fios, independente da variação da amplitude do movimento de flexo-extensão da articulação. Desta maneira, a sutura permaneceria com a mesma tensão diminuindo as chances de afrouxamento ou falha da mesma (COOK, 2010b). Apesar do trabalho realizado por Pelisson, Trapp e Kemper (2010) não ter verificado diferença na intensidade 
do movimento de gaveta utilizando-se pontos isométricos ou não em cadáveres no momento imediato após a sutura, não há estudos avaliando in vivo, em determinado momento pós- operatório, o afrouxamento ou falha da sutura sob as forças de tensão naturalmente exercidas no joelho.

Não houve complicações relacionadas ao fio em nenhum dos animais em que foram realizadas técnicas utilizando fio de náilon (monofilamentoso), e ocorreram infecções em dois animais onde foi utilizado o fio de poliéster (multifilamentoso). Vasseur (2007) estima um índice de infecção de 3 a $5 \%$ dos casos. Segundo Cook (2010a), os fios sintéticos multifilamentosos têm propriedades físicas superiores proporcionando maior rigidez, segurança do nó e suporte de carga, porém, os monofilamentosos são menos susceptíveis à infecção e reação tecidual. De maneira geral, o material de sutura multifilamentar, embora apresente maior resistência e mínima capacidade de estiramento, está associado a um grande aumento na incidência de infecção ou formação de fístulas (SMITH, 2000; HARASEN, 2010).

Doze joelhos receberam a técnica extracapsular nos pontos isométricos e fio poliéster com ou sem artrotomia, nos quais se observou uma melhora evidente na claudicação na maioria dos casos (Grau 1 e 2). Entretanto, em dois cães ocorreu reação ao fio e em outro houve ruptura do fio após 30 dias. Este animal foi submetido a uma segunda cirurgia. Em um estudo realizado em cães com peso acima de $30 \mathrm{~kg}$ que foram submetidos à técnica extracapsular com fio poliéster para estabilização do joelho, constatou-se que todos os cães recuperaram satisfatoriamente a função do membro, embora tenha havido leve progressão da artrose mesmo após a cirurgia (GUÉNÉGO et al., 2007).

O fio de poliéster, neste trabalho, demonstrouse mais predisposto para infecção, portanto é necessário ter cuidado com os fatores predisponentes e determinantes desta, tais como o tempo transoperatório, falhas na técnica asséptica, doenças concomitantes tais como endocrinopatias e diabetes mellitus, deficiência do sistema imune, terapia imunossupressora, condições gerais do animal e do local do sítio cirúrgico, tempo de anestesia, entre outros. Preferencialmente, deve-se minimizar os riscos com uma anti-sepsia copiosa, profilaxia antibiótica apropriada, fechamento de espaços mortos e redobrar os cuidado pós-operatório com a ferida cirúrgica (FOSSUM; WILLARD, 2008).

O resultado da cultura de bactérias de um dos animais em que houve reação inflamatória ao fio foi Staphylococcus spp., sensível somente à gentamicina. Este fato condiz com a literatura, que demonstra que o Staphylococcus spp. é a bactéria mais comumente encontrada em infecções pósoperatórias tanto da ruptura do ligamento cruzado cranial, assim como em todos os procedimentos cirúrgicos realizados na articulação do joelho (MARCHEVSKY; READ, 1999). No estudo realizado por Frey et al. (2010), constatou-se um índice de 4,2\% de infecção pós-operatória para a técnica extracapsular com fio de náilon. Entretanto, as variáveis, sexo, idade, raça, peso corporal e duração da cirurgia não foram considerados fatores predisponentes para a ocorrência de infecção. Dulish apud Tatarunas et al. (2007) associou a técnica extracapsular com fio multifilamentoso à infecção por Staphylococcus aureos e Micrococcus $s p p$., reação imunomediada e/ou reação alérgica ao material de sutura. Em outro estudo do mesmo autor, constatou-se um índice de $18 \%$ de complicações relacionadas à reação ao fio, onde foi concluído que as reações resultaram de suturas contaminadas.

Dos 28 casos, 18 apresentaram doenças ortopédicas concomitantes a ruptura do ligamento cruzado cranial, sendo que em 9, com a maioria sendo cães acima de $25 \mathrm{~kg}$, a afecção era displasia coxofemoral, e nos outros nove era luxação de patela medial, sendo em maior parte cães com peso abaixo de $20 \mathrm{~kg}$.

Todos os joelhos com luxação de patela receberam correção cirúrgica concomitante à 
do ligamento, e todos apresentaram melhora satisfatória da função do membro (Graus 0, 1 e 2). Observou-se que a correção da luxação de patela juntamente com a do ligamento cruzado cranial não influi no prognóstico, assim como demonstra o estudo de Costa et al. (2004), onde todos os animais que foram submetidos à correção para luxação de patela com ou sem a do ligamento, tiveram melhora clinica satisfatória. Cães de meia idade e com luxação de patela tem alto risco de sofrer ruptura do ligamento (CAMPBELL et al., 2010). Segundo Harasen (2006), a luxação de patela está altamente correlacionada com a ruptura do ligamento cruzado cranial, e estima-se um percentual de $20 \%$ de animais que apresentam a luxação sofrerão ruptura do cruzado cranial.

Dos cães com displasia coxofemoral, seis receberam a denervação concomitante a estabilização do joelho. Todos os cães que apresentaram displasia que foram submetidos à denervação coxofemoral concomitante à correção da ruptura do cruzado cranial melhoraram satisfatoriamente. Já os cães que não foram submetidos à denervação não obtiveram melhora clínica tão satisfatória. A presença de displasia coxofemoral influiu no prognóstico dos animais em que não foi realizada a denervação. Vale ressaltar a importância do diagnóstico e tratamento de doenças ortopédicas concomitantes à ruptura do ligamento cruzado cranial para uma correta avaliação do prognóstico para o paciente. Segundo Witsberger et al. (2008), as duas afecções concomitantes ocorrem principalmente em raças de grande porte, e clinicamente, são interdependentes, fato demonstrado neste trabalho. A média de idade desses cães foi de 5,5 anos, resultado que se aproxima da média de 5,1 anos relatada no estudo de Powers et al. (2005), relatando também uma prevalência de $32 \%$ de animais que apresentavam diagnostico inicial de displasia coxofemoral porém a principal causa da claudicação era na realidade a ruptura do ligamento cruzado cranial.

Dos 28 casos, 10 passaram por artrotomia concomitante à técnica extrarticular, sendo que todos tiveram melhora clínica satisfatória. Isso demonstra que a realização da artrotomia não influi na recuperação do animal em longo prazo. Chierichetti et al. (2001) relata que o tempo de recuperação em cães não artrotomizados é mais curto, o que se deve ao fato de que, com menor traumatismo cirúrgico, ocorre menor inflamação e consequentemente mais rápida reabilitação, porém não houve diferença significativa ao período final de avaliação com relação aos cães artrotomizados. Baseando-se neste fato, a realização da artrotomia em todos os casos é indicada, pois se estima que em 20 a 77\% dos casos há algum grau de lesão no menisco, sendo importante o diagnóstico e o tratamento no momento da cirurgia, reduzindo-se assim a morbidade do paciente e a necessidade de realizar cirurgias adicionais. Deve-se ressaltar que as lesões meniscais pós-cirurgicas muitas vezes são decorrentes de lesões já existentes, porém que não foram identificadas durante a cirurgia. Isso demonstra a importância da observação e inspeção cuidadosa do menisco (COOK, POZZI, 2010).

Dos 33 joelhos, foram realizados raios-X pré e pós operatório em 13, variando entre 30 dias a 2 anos e 141 dias entre o pré e pós cirúrgico. Pode-se observar que mesmo com a cirurgia para estabilização do joelho, independentemente da técnica adotada, houve progressão da artrose em todos os casos do período avaliado. Todos os animais exceto dois já apresentavam algum grau de doença articular degenerativa no momento do diagnóstico. A maioria dos cães apresentavam discreto movimento de gaveta (Grau 1), sendo que a maioria apresentou melhora clínica satisfatória (graus 0, 1 e 2). Não houve correlação entre progressão da artrose, intensidade de gaveta e grau de claudicação. Gordon et al. (2003) relata que a intensidade da artrose na radiografia não necessariamente pode estar relacionada com os sinais clínicos da função do membro.

Casos que não tiveram doenças ortopédicas concomitantes, nem acidentes por trauma durante a recuperação e nem reação ao fio, melhoraram 
clinicamente de maneira satisfatória independente da técnica adotada. Estudos demonstraram que apesar dos resultados clínicos satisfatórios proporcionados pelas diferentes técnicas utilizadas para a estabilização do joelho (VASSEUR, 2007), nenhuma delas previne a progressão da artrose ou tem diferença significativa na diminuição da progressão da mesma (MOORE; READ, 1996).

\section{Conclusão}

Pode-se concluir que a escolha das técnicas cirúrgicas para correção da ruptura do ligamento cruzado cranial: extracapsular com náilon, extracapsular nos pontos isométricos com náilon ou poliéster, Paatsama associada à técnica extracapsular com náilon e Paatsama, não influem no prognóstico, com relação à função do membro operado e que independentemente do tipo de técnica adotada, há melhora clínica pós-operatória satisfatória em $82 \%$ dos casos, apesar de persistência de discreto movimento de gaveta e progressão da artrose.

\section{Agradecimentos}

Agradeço aos funcionários do Hospital Veterinário da Universidade Estadual de Londrina e também aos proprietários dos animais que disponibilizaram seu tempo para ajudar na realização deste trabalho.

\section{Referências}

BENNETT, D. Joints and joint disease. In: WHITTICK, W. G. Canine orthopedics. 2. ed. Philadelphia: Lea \& Febiger, 1990. p. 776-779.

BUTTERWORTH, S. J.; DENNY, H. R. Joelhos. In:_ Cirurgia ortopédica em cães e gatos. São Paulo: Editora Roca, 2000. cap. 42, p. 396-427.

CAMPBELL, C. A.; HORSTMAN, C. L.; MASON, D. R.; EVANS, R. B. Severity of patellar luxation and frequency of concomitant cranial cruciate ligament rupture in dogs: 162 cases (2004-2007). Journal of the American Veterinary Medical Association, Schaumburg, v. 236, n. 8, p. 887-891, 2010.
CHIERICHETTI, A. L.; ALVARENGA, J.; PEDRO, C. R.; STOPLIGLIA, A. J. Ruptura de ligamento cruzado cranial. Estudo comparativo da técnica extra-articular com enxerto autógeno de fáscia lata com e sem artrotomia exploratória. Clínica Veterinária, São Paulo, n. 33, p. 3442, 2001.

CONZEMIUS, M. G.; EVANS, R. B.; BESANCON, M. F.; GORDON, W. J.; HORSTMAN, C. L.; HOEFLE, W. D.; NIEVES, M. A.; WAGNER, S. D. Effect of surgical technique on limb function after surgery for rupture of cranial cruciate ligament in dogs. Journal of the American Veterinary Medical Association, Schaumburg, v. 226, n. 2, p. 232-236, 2005.

COOK, J. L. Epidemiology of cranial cruciate ligament rupture. In: MUIR, P. Advances in the canine cranial cruciate ligament. Iowa: Wiley-Blackwell, 2010a. p. 9597.

Extracapsular stabilization. In: MUIR, P. Advances in the canine cranial cruciate ligament. Iowa: Wiley-Blackwell, 2010b. p. 163-168.

COOK, J. L; POZZI, A. Surgical treatment of concurrent meniscal injury. In: MUIR, P. Advances in the canine cranial cruciate ligament. Iowa: Wiley-Blackwell, 2010. p. 217-222.

COSTA, J. L. O.; PADILHA FILHO, J. G.; COSTA, C. M. B.; SHIMIZU F. L.; BORGES, L. H. A. Desmoplastia lateral estabilizadora e anti-rotacional com fáscia lata para correção de luxação medial de patela em cães. Revista Científica Eletrônica de Medicina Veterinária, Garça, n. 3, 2004. Disponível em: <http://www.revista. inf.br/veterinaria03/artigos/artigo02.pdf $>$. Acesso em: 23 abr. 2011.

FOSSUM, T. W.; WILLARD, M. D. Infecções cirúrgicas e seleção de antibióticos. In: FOSSUM, T. W. Cirurgia de pequenos animais. 3. ed. Rio de Janeiro: Elsevier, 2008. p. 79-89.

FREY, T. C.; HOELZLER, M. G.; SCAVELLI, T. D.; FULCHER, R. P.; BASTIAN, R. P. Risk factors for surgical site infection-inflammation in dogs undergoing surgery for rupture of the cranial cruciate ligament: 902 cases (2005-2006). Journal of the American Veterinary Medical Association, Schaumburg, v. 236, n. 1, p. 88-94, 2010.

GORDON, W. J.; CONZEMIUS, M. G.; RIEDESEL, E.; BESANCON, M. F.; EVANS, R.; WILKE, V.; RITTER, M. J. The relationship between limb function and radiographic osteoarthrosis in dogs with stifle osteoarthrosis. Veterinary Surgery, Philadelphia, v. 32, n. 5, p. 451-454, 2003. 
GUÉNÉGO, L.; ZAHRA, A; MADELÉNAT, A.; GAUTIER, R.; MARCELLIN-LITTLE, D. J.; HULSE, D. Cranial cruciate ligament rupture in large and giant dogs - A retrospective evaluation of a modified lateral extracapsular stabilization. Veterinary Comparative Orthopaedics Traumatology, Stuttgart, v. 20, n. 1, p. 4350, 2007.

HARASEN, G. A retrospective study of 165 cases of rupture of the canine cranial cruciate ligament. Canadian Veterinary Journal, Ottawa, v. 36, n. 4, p. 250-251, 1995.

Canine cranial cruciate ligament rupture in profile. Canadian Veterinary Journal, Ottawa, v. 44, n. 10, p. 846-854, 2003.

Patelar luxation. Canadian Veterinary Journal, Ottawa, v. 47, p. 817-818, 2006.

. What's new in orthopedics? Part 2. Canadian Veterinary Journal, Ottawa, v. 48, p. 1081-1082, 2007.

Walking the tightrope. Canadian Veterinary Journal, Ottawa, v. 51, p. 1167-1168, 2010.

HULSE, D. A. The stifle joint. In: OLMSTEAD, M. L. Small animal orthopedics. St. Louis: Mosby, 1995. p. 404-411.

JOHNSON, A. L. Fundamentos de cirurgia ortopédica e manejo de fraturas. In: FOSSUM, T. W. Cirurgia de pequenos animais. 3. ed. Rio de Janeiro: Elsevier, 2008. p. 930-1014.

JOHNSON, A. L.; JOHNSON, J. M. Cranial cruciate ligament rupture. Pathogenesis, diagnosis and postoperative rehabilitation. Veterinary clinical of North America: Small Animal Practice, Philadelphia, v. 23, p. 717-733, 1993.

MARCHEVSKY, A. M.; READ, R. A. Bacterial septic arthritis in 19 dogs. Australian Veterinary Journal, Brunswick, v. 77, n. 4, p. 233-237, 1999.

MOORE, K. W.; READ, R. A. Cranial cruciate ligament rupture in the dog. A retrospective study comparing surgical technique. Australian Veterinary Journal, Brunswick, v. 72, n. 8, p. 285-291, 1995.

Cranial cruciate ligament rupture in the $\operatorname{dog}-$ part II. Diagnosis and management. Compendium on Continuing Education for the Practicing Veterinarian, Lawrenceville, v. 18, n. 4, p. 381-391, 1996.
PELISSON, C. F.; TRAPP S. M.; KEMPER B. Estudo comparativo in vitro do movimento de gaveta em joelhos de caninos submetidos a duas técnicas extracapsulares de correção da instabilidade após ruptura do ligamento cruzado cranial. Ciência Rural, Santa Maria, v. 40, n. 6, p. 1335-1340, 2010.

POWERS, M. Y.; MARTINEZ, S. A.; LINCOLN, J. D.; TEMPLE, C. J.; ARNAIZ, A. Prevalence of cranial cruciate ligament rupture in a population of dogs with lameness previously attributed to hip dysplasia: 369 cases (1994-2003). Journal of the American Veterinary Medical Association, Schaumburg, v. 227, n. 7, p. 11091111, 2005.

SCHULZ, K. Ruptura do ligamento cruzado cranial. In: FOSSUM, T. W. Cirurgia de pequenos animais. 3. ed. Rio de Janeiro: Elsevier, 2008. p. 1254-1275.

SMITH, B. Viewpoints in surgery cruciate ligament rupture, extracapsular stabilization. Australian Veterinary Journal, Brunswick, v. 78, n. 6, p. 382-383, 2000.

TATARUNAS, A. C.; MATERA, J. M.; OLIVEIRA, R. M. D.; MACCHIONE, R. F.; BRUGNARO. M. Estudo retrospectivo do tratamento da ruptura de ligamento cruzado cranial no cão por técnica cirúrgica extraarticular. Brazilian Journal of Veterinary Research and Animal Science, São Paulo, n. 3, v. 44, p. 200-207, 2007.

VASSEUR, D. B. Articulação do joelho. In: SLATTER, D. Manual de cirurgia de pequenos animais. 3. ed. São Paulo: Manole, 2007. v. 2, p. 2090-2128.

VASSEUR, P. B.; BERRY, C. R. Progression of stifle osteoarthrosis following reconstruction of the cranial cruciate ligament in 21 dogs. Journal of the American Animal Hospital Association, Schaumburg, v. 28, p. 129136, 1992.

WHITEHAIR, J. G.;VASSEUR, P. B.; WILLITS, N. H. Epidemiology of cranial cruciate ligament rupture in dogs. Journal of the American Veterinary Medical Association, Schaumburg, v. 203, n. 7, p. 1016-1019, 1993.

WITSBERGER, T. H.; VILLAMIL, A.; SCHULTZ, L. G.; HAHN, A. W.; COOK, J. L. Prevalence of and risk factors for hip dysplasia and cranial cruciate ligament deficiency in dogs. Journal of the American Veterinary Medical Association, Schaumburg, v. 232, n. 12, p. 18181824, 2008. 
